\title{
Cometary Volatiles and the Origin of Comets
}

Michael F. A'Hearn ${ }^{1}$, Lori M. Feaga ${ }^{1}$, H. Uwe Keller ${ }^{2}$, Hideyo Kawakita ${ }^{3}$, Donald L. Hampton ${ }^{4}$, Jochen Kissel ${ }^{5}$, Kenneth P. Klaasen ${ }^{6}$, Lucy A. McFadden ${ }^{7}$, Karen J. Meech ${ }^{8}$, Peter H. Schultz ${ }^{9}$, Jessica M. Sunshine ${ }^{1}$, Peter C. Thomas ${ }^{10}$, Joseph Veverka ${ }^{10}$, Donald

K. Yeomans ${ }^{6}$, Sebastien Besse ${ }^{1}$, Dennis Bodewits ${ }^{1}$, Tony L. Farnham ${ }^{1}$, Olivier Groussin ${ }^{11}$, Michael S. Kelley ${ }^{1}$, Carey M. Lisse ${ }^{12}$, Frederic Merlin ${ }^{13}$, Silvia Protopapa1, Dennis D. Wellnitz ${ }^{ \pm}$

1Department of Astronomy, University of Maryland, College Park MD 20742, USA;

Institute for Geophysics and Extraterrestrial Physics, Technische Universität Braunschweig, 38106 Braunschweig, Germany

${ }^{3}$ Department of Physics, Kyoto Sangyo University, Kamigamo JP Kita-ku, Kyoto $603-$ 8555 Japan

${ }^{4}$ Geophysical Institute, University of Alaska Fairbanks, 903 Koyukuk Dr, Fairbanks, AK, 99775

${ }^{5}$ Max-Planck-Institut for Solar System Research, Max-Planck-Strasse 2, 37191 Katlenburg-Lindau, Germany (retired)

${ }^{6}$ Jet Propulsion Laboratory/Caltech, Pasadena, CA, 91109

${ }^{7}$ NASA Goddard Space Flight Center, Greenbelt MD 20771

Institute for Astronomy, 2680 Woodlawn Drive, Honolulu, HI 96822

${ }^{9}$ Department of Geological Sciences, Brown University, Providence, RI 02912 USA

${ }^{10}$ Department of Astronomy, Cornell University, Ithaca, NY, 14853

11 Laboratoire d'Astrophysique de Marseille, Université de Provence and CNRS, 13013 Marseille, France

12Space Department, JHU-APL, 11100 Johns Hopkins Road, Laurel, MD 20723

${ }^{13}$ LESIA, Observatoire de Paris, Université Paris 7, Batiment 17, 5 place Jules Janssen, Meudon Principal Cedex 92195, France.

\section{ABSTRACT}

We describe recent results on the $\mathrm{CO} / \mathrm{CO}_{2} / \mathrm{H}_{2} \mathrm{O}$ composition of comets and compare these with models of the protoplanetary disk. We argue that the cometary observations require reactions on grain surfaces to convert $\mathrm{CO}$ to $\mathrm{CO}_{2}$ and also require formation between the $\mathrm{CO}$ and $\mathrm{CO}_{2}$ snow lines. This then requires very early mixing of cometesimals in the protoplanetary disk analogous to the mixing described for the asteroid belt by Walsh and Morbidelli (2011). We suggest that most comets formed in the region of the giant planets, the traditional source of the Oort-cloud comets but not of the Jupiter-family comets.

\section{INTRODUCTION}


Our current understanding of cometary dynamics (e.g., Rickman 2010) implies that Jupiter-family comets (JFCs) formed primarily beyond Neptune, while long-period comets (LPCs), coming to us via the Oort cloud, and likely the source of many Halleytype comets (HTCs), formed both in the region of the giant planets followed by direct ejection to the Oort cloud or formed anywhere out to beyond Neptune and reached the inner Oort cloud via the scattered disk. Recent results on the volatile composition of comets, both in situ (A'Hearn et al. 2011) and via remote sensing (Ootsubo et al. 2011) suggest a more complicated scenario in the earliest stages of cometary formation and evolution. All comets may have formed in the region of the giant planets. These results also call into question the traditional composition deduced for volatiles condensing from the gas phase, notably $\mathrm{H}_{2} \mathrm{O}, \mathrm{CO}_{2}$, and $\mathrm{CO}$, in the protoplanetary disk (e.g., Williams and Cieza 2011), although very recent results on the composition of icy grains in quiescent clouds suggest a resolution of some of these problems (Noble et al. 2011).

Belton and Melosh (2009) have argued that the heterogeneity of the abundances of volatiles in JFCs should be explained as a result of evolution in the inner solar system. Here we explore the alternative, namely that the heterogeneity is largely a result of the original formation process. A similar conclusion has been reached in other recent investigations that will be discussed below.

\section{COMETARY VOLATILES}

The relative abundances of $\mathrm{H}_{2} \mathrm{O}, \mathrm{CO}_{2}$, and $\mathrm{CO}$ in cometary outgassing have been the subject of very limited studies due to the difficulty of the observations, particularly for $\mathrm{CO}_{2}$, which can only be observed beyond Earth's atmosphere. The data are summarized in Table 1 where we include some dynamical parameters together with the relevant ratios of abundances. The first measurements of all three species were for comet $1 \mathrm{P} / \mathrm{Halley}$ from the in situ mass spectrometers. Those data have had a variety of interpretations but the abundances we use here are the ones found by Rubin et al. (2011), who do not require an extended source for the $\mathrm{CO}$. Subsequent measurements included an indirect measurement via prompt emission in the ultraviolet Cameron bands of $\mathrm{CO}$ and direct detections with ISO. These measurements have been discussed by Bockelée-Morvan et al. (2004). More recent results include measurements from the Deep Impact spacecraft of 9P/Tempel 1 (Feaga et al. 2007a, 2007b) and of 103P/Hartley 2 (A'Hearn et al. 2011, Feaga et al. 2011) coupled with measurements of CO in Hartley 2 using HST (Weaver et al. $2011 \mathrm{a}, \mathrm{b}$ ). Meanwhile, the Japanese AKARI satellite has been used to carry out a survey of the three species in about two dozen comets (Ootsubo et al. 2011), although in most cases the results for $\mathrm{CO}$ are only upper limits, which still provide interesting constraints. We do not include here several measurements of only $\mathrm{CO}$ without $\mathrm{CO}_{2}$.

Observations of the coma may be misleading and/or confusing due to the presence of volatiles, principally $\mathrm{H}_{2} \mathrm{O}$ in the present context, in the form of ice rather than gas, as seen close to the nucleus in Hartley 2 (A'Hearn et al 2011) and much further from the nucleus in the outburst ejecta of 17P/Holmes (Yang et al. 2009). If one observes with a sufficiently large aperture that all the ice has evaporated before reaching the limit of the field, the measurements of gaseous water should correctly reflect the total rate of release of water. A wealth of data suggests that the different volatile ices are not mixed at the 
microscopic level but at some macroscopic level, the scale of which is not known, although we note that the volatiles excavated by Deep Impact at the scale of tens of meters were in the same relative abundances as those in the ambient outgassing of that comet. For this paper, we use the total release of water, both as ice and as gas, as the relevant measure for abundance ratios. We consider only observations within $2.5 \mathrm{AU}$ of the sun, distances at which $\mathrm{H}_{2} \mathrm{O}$-ice is normally expected to entirely sublimate within the field of view of typical remote sensing instruments. This is also the distance inside which the production rates of $\mathrm{OH}$ (from $\mathrm{H}_{2} \mathrm{O}$ ) and $\mathrm{CO}$ were parallel in comet Hale-Bopp (C/1995 O1) (Biver et al. 1997). The increase of abundances of other volatiles relative to water at larger heliocentric distances is easily seen also in the multiple observations of individual comets in the complete dataset of Ootsubo et al. (2011, see also Table 1).

The abundance ratios for many comets are given in Table 1. We have considered only comets for which there are measurements or upper limits for $\mathrm{CO}_{2}$. This table includes measurements at large heliocentric distances. Ootsubo et al. (2011) have explored the variation with distance of observation and shown that the ratio $\left[\mathrm{CO}_{2} / \mathrm{H}_{2} \mathrm{O}\right]$ varies over a wide range, with a lower limit that is independent of heliocentric distance and an upper limit that increases systematically with distance. Here we are interested more in long-term evolutionary effects and in Figure 1 we have plotted $\left[\mathrm{CO}_{2} / \mathrm{H}_{2} \mathrm{O}\right]$ versus perihelion distance, a parameter correlated with evolution through the maximum heating around the orbit. There is no trend among the JFCs and among the HTCs/L.PCs, the lone object that might suggest a trend is Halley, at $0.58 \mathrm{AU}$ and the only HTC in the sample. Note that the range of values for JFCs completely encompasses the range for LPCS. Figure 2 shows that ratio for the LPCs + Halley (a much smaller sample), plotted against the reciprocal semi-major axis of the orbit on approach, a useful proxy for the number of perihelion passages in the inner solar system. Again there is no trend except for Halley itself being an outlier with respect to the LPCs. The lack of trends in these diagrams, particularly the lack of any pattern for LPCs relative to JFCs, suggests that a primordial explanation for the variation should be considered, even though evolutionary effects likely play some role. There is no way, with present data, to know whether the low values for Halley indicates a trend or is an artifact of small-number statistics.

Meanwhile, the ratio $\left[\mathrm{CO}_{2} / \mathrm{CO}\right]$ varies by much more than two orders of magnitude in the limited sample for which direct measurements are available. There is not much range among the LPCs and HTCs where it is directly measured, but the lower limits for some LPCs require that the range be well over one order of magnitude. There is some indication from the lower limits that perhaps $\left[\mathrm{CO}_{2} / \mathrm{CO}\right]$ might be highest for the dynamically new comets. The two JFCs for which both species are detected yield ratios more than two orders of magnitude apart and spanning the entire range (including lower limits) for LPCs and Halley. This does not seem consistent with depletion of the highest volatility species through perihelion passages, nor does it seem consistent with bondbreaking by cosmic rays while in the Oort cloud. This also suggests that the ratio may be primordial. Mumma and Chamley (2011) present results from near-infrared spectroscopy on $\left[\mathrm{CO} / \mathrm{H}_{2} \mathrm{O}\right.$ ] (their Figure 8 ) in a dozen comets. Although they do not have results for $\mathrm{CO}_{2}$, the range of $\left[\mathrm{CO} / \mathrm{H}_{2} \mathrm{O}\right]$ is well over an order of magnitude, with JFCs and LPCs overlapping in most of that range. This also suggests similar origins. 
When considering the abundance ratio one must ask whether the relevant ratio is the ratio of species leaving the nucleus as gas or the total ratio of species, whether gaseous or solid when leaving the nucleus. For some problems, such as studying the nongravitational accelerations or torques on the nucleus, the ratio of gases is relevant, but for bulk chemical composition the total amount leaving the nucleus is more relevant. If the nucleus is globally heterogeneous and the different volatiles are coming from fundamentally different areas within the nucleus, the measured abundances may average over different cometesimals. The fact that the excavation by Deep Impact at Tempel 1 led to volatile abundances in essentially the same proportion as in the ambient outgassing (A'Hearn et al. 2008) argues that at least to depths of tens of meters the ices are mixed (macroscopically, not microscopically). These results are very hard to explain in an evolutionary scenario and we consider the primordial scenario more likely and therefore worth studying in more detail.

\section{PRIMORDIAL ABUNDANCES AND THE PROTOPLANETARY DISK}

We first note that most traditional models of the protoplanetary disk predict abundance ratios $\mathrm{CO}_{2} / \mathrm{CO}<<1$ (typically as low as $10^{-4}$ ) reflecting gaseous relative abundances and direct condensation from the gas. However, recognition of much higher ratios in the ices of interstellar dark clouds has led Garrod and Pauly (2011) to theoretically consider reactions of $\mathrm{OH}$ with $\mathrm{CO}$ on the surface of icy grains that lead to comparable abundances of $\mathrm{CO}$ and $\mathrm{CO}_{2}$ in interstellar dark clouds. Noble et al. (2011) have obtained similar results in the laboratory. We conclude that these surface reactions are critical to the formation of comparable abundances of $\mathrm{CO}$ and $\mathrm{CO}_{2}$ in cometary nuclei.

We further suggest that the wide range of abundance ratios in comets implies that the comets formed generally between the $\mathrm{CO}_{2}$ sand $\mathrm{CO}$ snow lines in the disk. The location of these snow lines differs among models and even varies with the evolution of the disk for any single model. For some recent models of the protoplanetary disk, e.g., that by Dodson-Robertson et al. (2009), these two snow lines are in the region of the giant planets. It seems unlikely that inclusion of grain-surface reactions would have major effects on the thermal profile of the disk. Thus we suggest that most comets formed in the region of the giant planets, the traditional origin for Oort-cloud comets but not for JFCs.

It is widely understood that the giant planets migrated during the early stages of the solar system although ideas on the details of that migration are changing rapidly.

Recently Walsh and Morbidelli (2011), in order to explain the distribution of asteroidal orbital properties, have invoked migration of Jupiter and Satum beginning much earlier and much closer to their present-day orbital positions than in recently preceding dynamical models. This migration mixed asteroids both outward and inward as the direction of migration changed and ultimately mixed up most of the asteroids in the outer portions of the asteroid belt. We suggest that a similar process must have occurred for the comets, although the role of specific planets is not clear without more work. Some comets were ejected directly to the Oort cloud as classically understood, but many others must have been ejected to the scattered disk and thus provided a source for the JFCs. The details of this process require a detailed, quantitative simulation that is beyond the scope 
of this paper, one involving grain reaction rates as well as specific planetary migration rates since the time scale is short.

We note that such mixing could also explain heterogeneity within individual cometary nuclei since this very early planetary migration could have occurred while the cometesimals were still accreting into nuclei. A'Hearn et al. (2011) and Feaga et al. (2011) have discussed the variations in relative abundances in Hartley 2 as it rotates and this strongly suggests that the larger lobe of the nucleus has much less volatile content than the small lobe. It is not yet clear how the relative abundances of the three ices varies because a detailed, quantitative model of the sublimation of grains in the coma is required. Because this comet is in excited state rotation now and the observed changes in rotational state during the last apparition imply different rotational states earlier in its history, seasonal effects should be minimal, suggesting that the two lobes of the nucleus likely formed at different heliocentric distances. The obvious spatial heterogeneity in the outgassing of Tempel I (Feaga et al. 2007a), on the other hand, could be either a seasonal evolutionary effect or a primordial effect. A variety of other earlier measurements by remote sensing have reported chemical changes with rotation suggesting that other comets also have nuclear heterogeneity, although in those cases it is not possible to assess the relative importance of primordial abundances, seasonal effects, and long-term evolutionary effects.

Mixing of this sort also appears to be needed to explain the $\mathrm{D} / \mathrm{H}$ ratio of comet Hartley 2 as pointed out by Hartogh et al. (2011). They measured the ratio to be equal to SMOW (Standard Mean Ocean Water), i.e.,, a factor two less than in the few LPCS + Halley for which it is measured. This suggests that at least Hartley 2 was formed in a region with $\mathrm{D} / \mathrm{H}$ similar to that on Earth. If JFCs formed in the Kuiper belt and LPCs formed in the region of the giant planets, the differences should go in the opposite direction according to Hartogh et al. Furthermore, Hogerheijde et al. (2011) have argued that the ortho-para ratio of water in a protoplanetary disk, which is much lower than that in comets in our own solar system, also requires comets to contain material that condensed in a wide variety of locations in the protoplanetary disk.

\section{SUMMARY}

Observations of the abundances of the volatile species $\mathrm{H}_{2} \mathrm{O}, \mathrm{CO}_{2}$, and $\mathrm{CO}$ in comets show a wide range of abundance ratios in both JFCs and LPCs/HTCs. This seems unlikely to be the result of evolution through successive perihelion passages. We suggest that both classes of comets, or at least the cometesimals that might later have assembled, formed between the $\mathrm{CO}_{2}$ and $\mathrm{CO}$ snow lines, thus allowing the wide range of abundance ratios. They were subsequently scattered by planetary migration to lead to the present dynamical types of comets, much as the asteroids were mixed up in the dynamical model of Walsh and Morbidelli (2011). This could also explain variations within individual nuclei since this migration takes place earlier than previously modeled and is relatively fast.

\section{REFERENCES}

A Hearn, M. F. et 33 al. 2011. Science 332, 1396. A'Hearn, M. F., et 9 al. 2008. Earth,Plan.Space, 60,61. 
Belton, M. J. S. and Melosh, J., 2009, Icarus 200, 280.

Biver, N., et 22 al., 1997, Earth,Moon,Planets, 78, 5.

Bockelée-Morvan, D., Crovisier, J., Mumma, M. J., and Weaver, H. A., 2004, In Comets II, ed. M. Festou et al. (Tucson, UAz.) 391.

Crovisier, J., Leech, K., and Bockelee-Morvan, D, 1997, Science, 275, 1905.

Dodson-Robinson, S. E., Willacy, K., Bodenheimer, P., Turner, N. J., and Beichman, C. A., 2009, Icarus, 200, 672

Feaga, L. M., A'Hearn, M. F., Sunshine, J. M., Groussin, O., and Farnham, T. L., 2007a, Icarus, $190,345$.

Feaga, L., Groussin, O., Sunshine, J., and A'Hearn, M., 2007b, BAAS, 39, 450.

Feaga, L. M., et 7 al., 2011, DPS-EPSC 2011, 1342.

Garrod, R T and Pauly, T., 2011, ApJ, 735, 15.

Hartogh, P. et 12 al., 2011, Nature, 478, 218.

Hogerheijde, M. R., et 14 al. 2011, Science, 334, 338.

Mumma, M. J., and Charnley, S. B., 2011, ARAA, 49, 471.

Noble, J. A., Dulieu, F., Congiu, Eu, and Fraser, H. J., 2011, ApJ, 735, 121.

Ootsubo, T., et 13 al, 2011, Ap), submitted.

Rickman, H., 2010, Lect.NotesPhys., 790, 341.

Rubin, M., Tenishev, V. M., Combi, M. R., Hansen, K. C., Gombosi, T. I., Altwegg, K., and Balsiger, H., 2011, Icarus, 213, 655.

Walsh, K. J., and Morbidelli, A., 2011, A\&A, 735, 15.

Weaver, H. A., Feldman, P. D., A'Hearn, M. F., Dello Russo, N., and Stern, S. A., 2011a

AplLett, 734, L5.

Weaver, H. A., Feldman, P. D., A'Hearn, M. F., Dello Russo, N., and Stern, S. A., 2011b, EPSC-DPS 2011, 1057.

Williams, J. P., and Cieza, L. A., 2011, ARAA, 49,67.

Yang, B., Jewitt, D., and Bus, S. J., 2009, AJ, 137, 4538.

\section{FIGURE CAPTIONS}

Figure 1. Relative Production of $\mathrm{CO}_{2}$ as a function of perihelion distance logarithmic scales. JFCs show no trend with perihelion distance and span more than the entire range of relative production rates by LPCs. The point in the lower left of the diagram is $1 \mathrm{P} /$ Halley, the only HTC in the sample.

Figure 2. Relative production of $\mathrm{CO}_{2}$ as a function of original reciprocal semi-major axis for LPCS and HTCs - logarithmic scales. 1P/Halley is the unusually low point and is the only HTC in the sample. 
Table 1. Abundance Ratios of Volatiles

\begin{tabular}{|c|c|c|c|c|c|c|c|c|c|c|}
\hline Object & $\mathrm{R}_{\mathrm{k}}$ & q & $(1 / a)_{0}$ & $\begin{array}{l}\mathrm{Q}\left(\mathrm{CO}_{2}\right) / \\
\mathrm{Q}\left(\mathrm{H}_{2} \mathrm{O}\right)\end{array}$ & $\sigma$ & $\begin{array}{l}\mathrm{Q}(\mathrm{CO}) / \\
\mathrm{Q}\left(\mathrm{H}_{2} \mathrm{O}\right)\end{array}$ & $\sigma$ & $\begin{array}{c}\mathrm{Q}\left(\mathrm{CO}_{2}\right) / \\
\mathrm{Q}(\mathrm{CO})\end{array}$ & $\sigma$ & Ref \\
\hline & {$[\mathrm{AU}]$} & {$[\mathrm{AU}]$} & {$\left[10^{-6} \mathrm{AU}^{-1}\right]$} & & & & & & & \\
\hline 19P/Borrelly & 2.19 & 1.36 & & 0.241 & 0.009 & $<0.168$ & & $>1.44$ & & 1 \\
\hline $22 \mathrm{P} / \mathrm{Kopff}$ & 1.61 & 1.59 & & 0.201 & 0.002 & $<0.024$ & & $>8.38$ & & 1 \\
\hline$"$ & 1.61 & & & 0.193 & 0.001 & $<0.019$ & & $>10.2$ & & 1 \\
\hline$"$ & 2.42 & & & 0.104 & 0.005 & $<0.149$ & & $>0.70$ & & 1 \\
\hline$"$ & 2.43 & & & 0.052 & 0.005 & $<0.169$ & & $>0.31$ & & 1 \\
\hline " & 2.43 & & & 0.039 & 0.003 & $<0.105$ & & $>0.37$ & & 1 \\
\hline $29 \mathrm{P} / \mathrm{S}-\mathrm{W} 1$ & 6.18 & 5.72 & & $<0.025$ & & 4.716 & 0.327 & $<0.005$ & & 1 \\
\hline${ }^{\prime \prime}$ & 6.18 & & & $<0.021$ & & 4.628 & 0.269 & $<0.005$ & & 1 \\
\hline 64P/Swift-Gehrels & 2.27 & 1.35 & & 0.326 & 0.017 & $<0.273$ & & $>1.19$ & & 1 \\
\hline 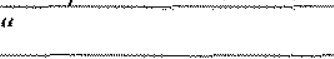 & 2.27 & & & 0.255 & 0.010 & $<0.205$ & & $>1.24$ & & 1 \\
\hline 67P/Chur-Ger & 1.84 & 1.29 & & 0.070 & 0.003 & $<0.127$ & & $>0.55$ & & 1 \\
\hline 81P/Wild 2 & 1.74 & 1.59 & & 0.177 & 0.002 & $<0.026$ & & $>6.81$ & & 1 \\
\hline$“$ & 1.74 & & & 0.118 & 0.001 & $<0.019$ & & $>6.21$ & & 1 \\
\hline$"$ & 1.74 & & & 0.144 & 0.001 & $<0,23$ & & $>6.26$ & & 1 \\
\hline $88 \mathrm{P} /$ Howell & 1.74 & 1.41 & & 0.242 & 0.002 & $<0.025$ & & $>19.7$ & & 1 \\
\hline$"$ & 1.73 & & & 0.258 & 0.002 & $<0.025$ & & $>10.32$ & & 1 \\
\hline $116 \mathrm{P} /$ Wild 4 & 2.22 & 2.17 & & 0.104 & 0.004 & $<0.121$ & & $>0.86$ & & 1 \\
\hline$"$ & 2.22 & & & 0.070 & 0.002 & $<0.092$ & & $>0.76$ & & 1 \\
\hline $118 \mathrm{P} / \mathrm{S}-\mathrm{L} 4$ & 2.18 & 2.01 & & 0.153 & 0.004 & $<0.097$ & & $>1.58$ & & 1 \\
\hline$"$ & 2.18 & & & 0.292 & 0.008 & $<0.137$ & & $>2.13$ & & 1 \\
\hline 144P/Kushida & 1.70 & 1.43 & & 0.147 & 0.001 & $<0.022$ & & $>6.68$ & & 1 \\
\hline 4 & 1.70 & & & 0.159 & 0.002 & $<0.014$ & & $>11.36$ & & 1 \\
\hline
\end{tabular}




\begin{tabular}{|c|c|c|c|c|c|c|c|c|c|c|}
\hline 157P/Tritton & 1.48 & 1.43 & & 0.071 & 0.003 & $<0.094$ & & $>0.76$ & & 1 \\
\hline$"$ & 1.48 & & & 0.118 & 0.004 & $<0.126$ & & $>0.94$ & & 1 \\
\hline C/Broughton (2006 F2) & 2.43 & 2.43 & 15 & 0.235 & 0.003 & $<0.025$ & & $>9.4$ & & 1 \\
\hline$"$ & 3.20 & & & 0.588 & 0.027 & $<0.207$ & & $>2.8$ & & 1 \\
\hline C/McNaught (2006 Q1) & 2.78 & 2.76 & 34 & 0.446 & 0.007 & $<0.058$ & & $>7.7$ & & 1 \\
\hline 4 & 3.64 & & & 0.513 & 0.036 & $<0.359$ & & $>1.4$ & & 1 \\
\hline C/Christensen (2006 W3) & 3.66 & 3.13 & 378 & 1.020 & 0.026 & 3.659 & 0.096 & 0.279 & 0.002 & 1 \\
\hline$"$ & 3.13 & & & 0.421 & 0.006 & 1.037 & 0.018 & 0.41 & 0.005 & 1 \\
\hline C/LINEAR (2007 G1) & 2.80 & 2.65 & 245 & 0.227 & 0.006 & $<0.119$ & & $>1.9$ & & 1 \\
\hline C/Lulin $(2007 \mathrm{~N} 3)$ & 1.28 & 1.21 & 30 & 0.119 & 0.001 & $<0.027$ & & $>4.4$ & & 1 \\
\hline$"$ & 1.70 & & & 0.100 & 0.001 & $<0.014$ & & $\geq 7.1$ & & 1 \\
\hline C/Siding Spring (2007 Q3) & 3.29 & 2.25 & 34 & 0.185 & 0.004 & $<0.080$ & & $>2.3$ & & 1 \\
\hline C/Garrad (2008 Q3) & 1.81 & 1.80 & 246 & 0.277 & 0.002 & 0.321 & 0.007 & 0.863 & 0.018 & 1 \\
\hline tt & 1.81 & & & 0.248 & 0.002 & 0.267 & 0.006 & 0.929 & 0.021 & 1 \\
\hline$"$ & 2.96 & & & 0.702 & 0.053 & $<0.517$ & & $>1.4$ & & 1 \\
\hline 1P/Halley & 0.86 & 0.58 & 54171 & 0.025 & 0.004 & 0.13 & 0.018 & 0.192 & 0.27 & 2 \\
\hline C/Hale-Bopp (1995 01) & 4.28 & 0.91 & 3800 & $>0.12$ & & & & $>0.14$ & & 3 \\
\hline " & 2.96 & & & 0.190 & 0.081 & 0.59 & 0.25 & 0.32 & 0.14 & 3 \\
\hline 9P/Tempel 1 & 1.49 & 1.49 & & 0.07 & 0.02 & 0.11 & 0.05 & 0.64 & 0.29 & 4,5 \\
\hline 103P/Hartley 2 & 1.06 & 1.04 & & 0.20 & 0.06 & 0.002 & 0.001 & 100 & 45 & 6,7 \\
\hline
\end{tabular}

Note: Columns: 1. Object name and ID; 2. Heliocentric distance at time of observation; 3 . Perihelion distance at observed apparition; 4. Reciprocal semi-major axis on approach to planetary region; 5-10. Ratios of production rates with errors or limits as appropriate; 11 . Reference for data.

Abbreviated Names: $1 . \mathrm{S}-\mathrm{W} 1=$ Schwassmann-Wachmann 1; 2. Chur-Ger $=$ Churyumov-Gerasimenko; 3. S-L $4=$ ShoemakerLevy 4

References: 1. Ootsubo et al. 2011; 2. Rubin et al. 2011; 3. Crovisier et al. 1997; 4. Feaga et al. 2007a; 5. Feaga et al. 2007b; 6. A'Hearn et al. 2011; 7. Weaver et al. 2011 


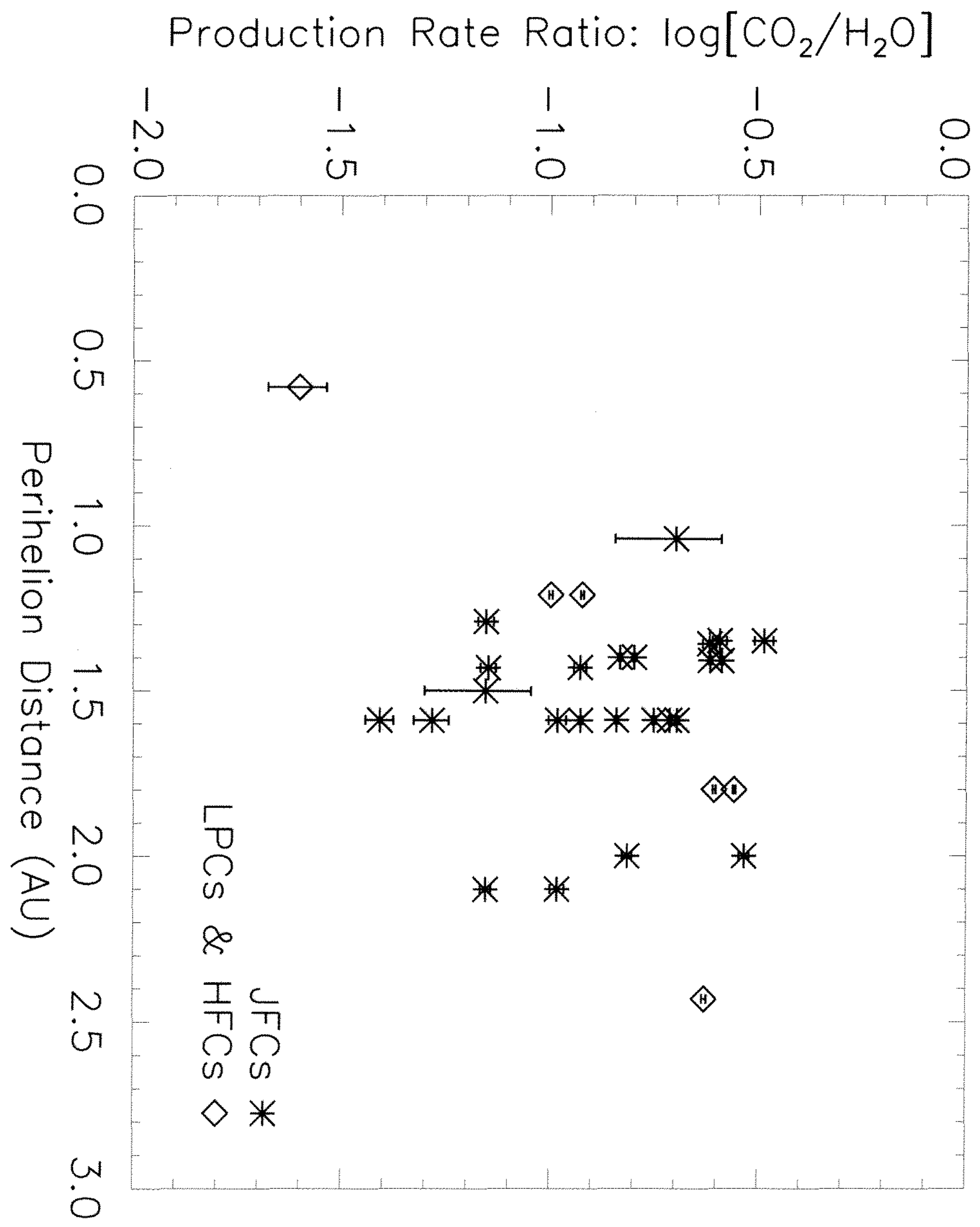




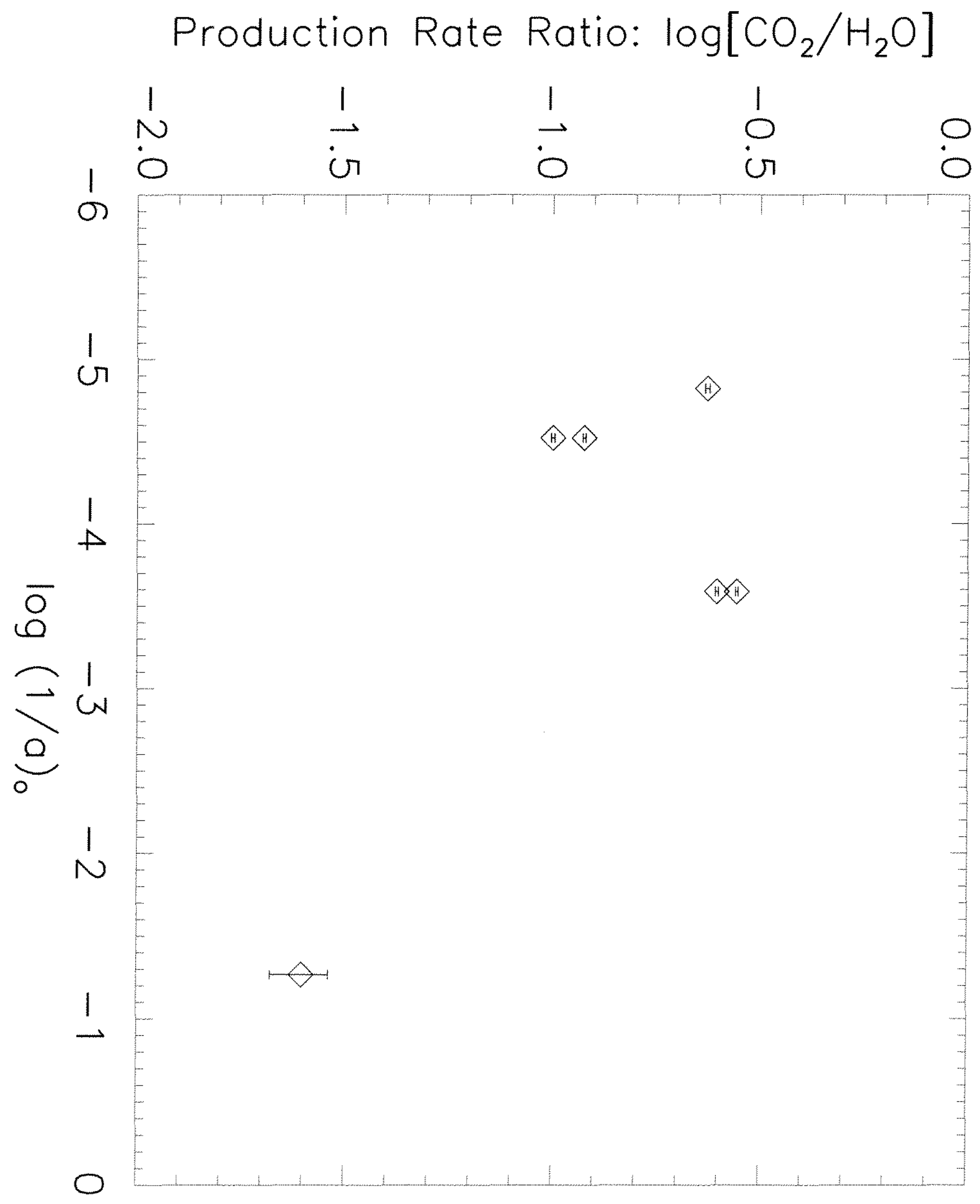

